\title{
NACT + IMRT versus NACT + IMRT + CCRT in locoregionally advanced NPC patients: a retrospective study
}

This article was published in the following Dove Press journal:

OncoTargets and Therapy

\author{
Xialin Chen ${ }^{1,2}$ \\ Xiang Zhu ${ }^{3}$ \\ Jianfang Wang ${ }^{1,2}$ \\ Jianjiang Liu ${ }^{1,2}$ \\ Rong $\mathrm{Ji}^{4}$
}

'Department of Oncology, Shaoxing People's Hospital, Shaoxing, Zhejiang 3।2000, China; ${ }^{2}$ Department of Oncology, Shaoxing Hospital of Zhejiang University, Shaoxing, Zhejiang 3 I2000, China; ${ }^{3}$ Department of Radiation Oncology, Zhejiang Cancer Hospital, Hangzhou, Zhejiang 3I0022, China; ${ }^{4}$ Department of Radiation Oncology, Shaoxing Second Hospital, Shaoxing, Zhejiang $3 \mid 2000$, China
Correspondence: Rong Ji

Department of Radiation Oncology, Shaoxing Second Hospital, No 123 ,

Yan'an Road, Shaoxing, Zhejiang 312000 ,

China

Email jirongsxsh@yeah.net
Purpose: The outcomes and safety profiles of neoadjuvant chemotherapy (NACT) + intensity modulated radiotherapy (IMRT) or NACT + IMRT + concurrent chemoradiotherapy (CCRT) in locoregionally advanced nasopharyngeal carcinoma (NPC) patients were retrospectively analyzed.

Patients and methods: Between 2010 and 2014, 125 patients with stage III-IVb NPC, who were treated with IMRT $(36,28.8 \%)$ or IMRT + CCRT $(89,71.2 \%)$ following NACT, participated in the research. There were grade 3-4 toxicities during NACT or radiotherapy (RT) in NACT + IMRT group and NACT + IMRT + CCRT group.

Results: MRI within 3 months demonstrated that no patient suffered with progressive disease, 116 patients $(92.8 \%)$ achieved a response rate (RR) with the complete response (CR) rate of $70.4 \%$ (88/125) and partial response (PR) rate of 22.4\% (28/125), and nine patients (7.2\%) showed stable disease (SD) at the primary site and metastatic nodes. Compared with NACT + IMRT group, patients in NACT + IMRT + CCRT group did not show significantly better RR (93.3\% vs $91.7 \%, P=1.00)$, CR rate $(71.9 \%$ vs $66.7 \%, P=0.67)$, or PR rate $(21.4 \%$ vs $25 \%$, $P=0.81$ ). There was no significant difference in overall survival (OS, $P=0.114$ ), local relapse-free survival (LRFS, $P=0.124$ ), distant metastasis-free survival (DMFS, $P=0.668$ ) or progressionfree survival (PFS, $P=0.475$ ) between NACT + IMRT group and NACT + IMRT + CCRT group. T classification $(P=0.042)$ and $\mathrm{N}$ classification $(P=0.021)$ were independent prognostic factors for DMFS.

Conclusion: To sum up, no significant difference was observed in combined RR, CR rate, LRFS, DMFS, PFS, or OS between the two groups.

Keywords: advanced nasopharyngeal carcinoma, intensity modulated radiotherapy, concurrent chemoradiotherapy, neoadjuvant chemotherapy

\section{Introduction}

Nasopharyngeal carcinoma (NPC), which is endemic in South China, is the eighth most common cause of cancer-related mortality. ${ }^{1}$ At initial diagnosis, the majority of patients (75\%-90\%) suffered with locoregionally advanced NPC. ${ }^{2}$ The standard treatment for locoregionally advanced NPC is cisplatin (CDDP)-based chemoradiotherapy (CRT) according to randomized trials ${ }^{3,4}$ and meta-analyses. ${ }^{5,6}$

Intensity modulated radiotherapy (IMRT), which yields more satisfactory outcomes than conventional radiotherapy $(\mathrm{RT}),{ }^{7,8}$ delivers a higher radiation dose to the tumor tissues while limiting the dose to normal tissues in NPC patients. Although IMRT has greatly improved overall survival (OS) of NPC patients, more than $10 \%$ patients still 
faced with a high risk of developing distant metastasis after IMRT, ${ }^{9}$ indicating IMRT was not sufficient for treatment of NPC patients.

Chemotherapy was important to control metastasis of NPC patients, ${ }^{4}$ but the efficacy of concurrent CRT (CCRT) in combination with IMRT was controversial. Retrospective studies in patients with T4 NPC demonstrated that CCRT + IMRT failed to improve patients' OS, distant metastasis-free survival (DMFS), or progression-free survival (PFS) compared to IMRT. ${ }^{10}$ In another study of stage II-IVb NPC patients, adding CCRT showed significant improvement of DFS. ${ }^{11}$

Although neoadjuvant chemotherapy (NACT) failed to improve OS in previous Phase III trials, certain Phase II studies have reported promising results. ${ }^{12,13}$ Hui et al reported that in the NACT group, patients' 3-year OS rate was $94.1 \%$, which was significantly higher than that of the CDDP-based CRT group (67.7\%). ${ }^{14}$ A meta-analysis demonstrated that NACT resulted in OS gain of 5.13\% and reduced the distant metastasis rate within 3 years with no significant reduction in the locoregional recurrence rate. ${ }^{15}$ Therefore, the value of NACT in locoregionally advanced NPC remains controversial. Novel strategies are required to effectively improve patients' OS.

The effectiveness and safety of NACT regimen (GEM + CDDP/carboplatin) followed by CDDP-based CRT have been confirmed, which demonstrated that CDDP-based CRT induced high frequencies of hematologic toxicities and nausea/vomiting. ${ }^{13}$ Nedaplatin (NDP), a platinum complex with a similar action mechanism but different molecular structure from CDDP, was evidenced to be at least as effective as CDDP in the treatment of locoregionally advanced NPC. ${ }^{16}$ CCRT was regarded as standard therapeutic approach for locoregionally advanced NPC. ${ }^{17}$

The aim of the present retrospective study was to compare the efficacy of NACT regimen (GEM + NDP) + IMRT and NACT regimen $($ GEM + NDP) + IMRT + CCRT (NDP), to determine the necessity of CCRT following NACT in the era of IMRT.

\section{Patients and methods}

\section{Patient eligibility}

Between September, 2010 and December, 2014, 125 eligible NPC patients, who suffered from WHO type II (nonkeratinizing differentiated carcinoma) or type III (nonkeratinizing undifferentiated carcinoma) $\mathrm{NPC}^{18}$ and stage III-IVB NPC (2010 American Joint Committee on Cancer [AJCC] staging system for NPC) $)^{19}$ and whose Karnofsky Performance Scale score was $\geq 70$ with no distant metastasis, adequate hepatic, renal or hematologic function, were treated at Zhejiang Cancer Hospital and participated in our study.

Prior inform and written consent was obtained from each patient. The current study was approved by the Ethics Committee of Zhejiang Cancer Hospital. The research was carried out in accordance with the Helsinki Declaration. Our report complies with all IRB requirements at the institution(s) where the work was performed.

\section{Physical examination}

Initial evaluation, which was carried out before the treatment in NPC patients, included complete blood cell count, comprehensive serum chemistry profile, chest X-ray or computed tomography (CT), electrocardiogram, MRI scans of the nasopharynx and upper neck, nasopharyngoscopy, and liver ultrasonography.

\section{Chemotherapy}

The NACT regimen was carried out as follows: NDP $\left(25 \mathrm{mg} / \mathrm{m}^{2}\right.$, day $1-3,60$ minutes, every 3 weeks) and GEM $\left(1,000 \mathrm{mg} / \mathrm{m}^{2}\right.$, day 1 and 5, 30 minutes, every 3 weeks $)$. In case of low blood count or intolerable toxicity, which was resulted from the preceding chemotherapy cycle, the dosage would be modified accordingly. If the count of platelet, leukocyte, or red blood cell decreased to $\leq 25 \times 10^{9} / \mathrm{L}, \leq 1 \times 10^{9} / \mathrm{L}$ or $\leq 50 \times 10^{9} / \mathrm{L}$, respectively, the dosage would decrease by $25 \%$ in the next cycle.

CCRT which was carried out as follows: NDP $\left(25 \mathrm{mg} / \mathrm{m}^{2}\right.$, day 1-3 at every 3 weeks, 60 minutes during IMRT) would be postponed even suspended when the grade 4 hematologic adverse events or intolerable toxicities arose.

\section{RT}

The gross tumor volume (GTV) was defined as the primary tumor (including lymph nodes). The planning target volume of the primary tumor (PTVg) was defined as the GTV of the tumor with a $0.3-0.5 \mathrm{~cm}$ margin, while the planning target volume of the metastatic nodes (PTVnd) was defined as the GTV of the nodes with a $0.3-0.5 \mathrm{~cm}$ margin. A total dose of 69 and 63 Gy over 6 weeks in 30 fractions was prescribed for PTVg and GTVnd, respectively.

The clinical target volume (CTV) was defined as the anterior third of the clivus, the entire nasopharyngeal cavity, parapharyngeal space, pterygoid plates, posterior third of the nasal cavity, maxillary sinus, inferior sphenoid sinus, and the drainage of the upper neck (levels II, III, and Va) in N0. Levels IV and Vb were defined as N1-3. PTVH was 
Table I Mean dose and maximum dose of crucial OAR

\begin{tabular}{l|l|l}
\hline Dose (Gy) & Mean dose & Maximum dose \\
\hline OAR & $23.4(18.3-27.5)$ & $52.5(49.5-56.8)$ \\
\hline Brainstem & $21.6(14.9-29.8)$ & $34.2(28.9-42.6)$ \\
Spinal cord & $30.5(22.9-39.6)$ & $45.2(39.2-51.3)$ \\
Optic chiasm & $28.7(20.3-39.8)$ & $47.9(40.1-55.3)$ \\
Left optic nerve & $32.2(15.6-52.2)$ & $50.2(41.4-58.7)$ \\
Right optic nerve & $20.5(13.2-24.6)$ & $58.4(46.8-68.6)$ \\
Left temporal lobe & $21.2(12.6-26.8)$ & $60.7(48.7-69.8)$ \\
Right temporal lobe & $31.6(24.3-36.5)$ & $60.1(50.2-68.5)$ \\
Left parotid gland & $30.2(25.7-37.6)$ & $58.5(54.2-62.7)$ \\
Right parotid gland & $34.5(28.2-42.5)$ & $47.4(39.5-55.3)$ \\
Left TMJ & $35.7(31.5-40.6)$ & $45.2(36.2-56.4)$ \\
Right TMJ &
\end{tabular}

Abbreviations: OAR, organ at risk; TMJ, temporomandibular joint.

defined as the high-risk CTV with a $0.3-0.5 \mathrm{~cm}$ margin, while PTVL was defined as the low-risk CTV. A total dose of 60 and 54 Gy in 30 fractions was prescribed for PTVH and PTVL, respectively.

Patients received IMRT with 6 MV X-rays. All patients were irradiated with one fraction daily for 5 days/week.

The mean dose and maximum dose (in Gy) to crucial organ at risk, including brainstem, spinal cord, optic chiasm, left/right optic nerve, left/right temporal lobe, left/right parotic gland, and left/right temporomandibular joint, were exhibited in Table 1.

The dose-volume histogram statistics of targets, including PGTVnx, GTVnd, PTVnd, PTV1, and PTV2, are presented in Table 2.

The contouring of GTV was closely corresponding to the original diseased tissues in all dimensions when considering the imaging post $\mathrm{NACT} .{ }^{20}$

\section{Treatment response, follow-up, and adverse effects}

Treatment-related toxicities were graded according to the National Cancer Institute Common Toxicity Criteria, version 3.0. ${ }^{21}$

PFS was estimated from the date of pathological diagnosis to the date of relapse or metastasis following the completion of all treatments. MFS and RFS were defined as the interval from the date of pathological diagnosis to the date of distant metastasis or relapse. OS was measured from the date of pathological diagnosis to the date of death or the last date when the patient was recorded alive. Prior to treatment and after RT within 3 months, a follow-up MRI of the nasopharynx and upper neck and intensive CT of chest and abdomen were carried out to evaluate responses based on the Response Evaluation Criteria in Solid Tumors criteria. ${ }^{22}$

After the completion of the treatments, patients were required to attend outpatient checkups every 3 months in the subsequent 2 years followed by attention of outpatient checkups every 6 months while the patients remained alive. Each follow-up included clinical examination, nasopharyngoscopy, MRI, intensive CT of the chest and abdomen, comprehensive serum chemistry profiles, and blood cell count. Other tests were performed at the discretion of the treating physician.

\section{Statistical analysis}

Descriptive statistical analyses were applied to characterize the patients in NACT + IMRT and NACT + IMRT + CCRT groups. Chi-squared test, which was carried out with SPSS 19.0 software (IBM Corporation, Armonk, NY, USA), was adopted for the estimation of the differences in clinical characteristics (WHO histology, AJCC stage, T stage, N stage, histologic types, and course of NACT) and demographic variables (gender, age, alcohol use, smoking history, and family history). Survival rates were calculated by the Kaplan-Meier method. Univariate analysis with log-rank test and multivariate analysis by the Cox proportional hazard model were done to test the independent prognostic factors for patients' OS, DMFS, local relapse-free survival (LRFS), and PFS. Statistical significance was set at $P<0.05$.

\section{Results}

\section{Patients}

Characteristics of NPC patients treated with NACT + IMRT or NACT + IMRT + CCRT were summarized and exhibited

Table 2 DVH statistics of targets

\begin{tabular}{|c|c|c|c|c|c|}
\hline & \multicolumn{5}{|l|}{ Average (range) } \\
\hline & PGTVnx & GTVnd & PTVnd & PTVI & PTV2 \\
\hline Volume $\left(\mathrm{cm}^{3}\right)$ & $60.1(18.2-156.4)$ & $20.5(6.5-55.9)$ & $63.4(\mid 4.7-144.3)$ & $320.6(212.6-598.7)$ & I $72.4(78.3-305.7)$ \\
\hline Minimum dose (Gy) & $62.1(60.2-63.5)$ & $61.8(60.7-63.6)$ & $50.4(45.8-57.6)$ & $48.7(45.3-52.8)$ & $40.7(37.1-44.7)$ \\
\hline Maximum dose (Gy) & $72.6(71.5-73.2)$ & $70.8(69.2-73.1)$ & $66.8(63.7-69.2)$ & $71.3(69.8-73.6)$ & $70.2(67.8-73.4)$ \\
\hline Mean dose (Gy) & 70.2 (68.2-72.2) & $65.2(63.4-68.4)$ & 63.9 (6I.7-68.7) & $67.2(64.5-69.6)$ & $60.4(58.4-62.6)$ \\
\hline
\end{tabular}

Abbreviations: DVH, dose-volume histogram; GTV, gross tumor volume; PTV, planning target volume; PTVnd, planning target volume of the metastatic nodes. 
in Table 3. The median age of diagnosis was 55 years (17-77 years) in NPC patients. In total, $83(66.4 \%)$ of the patients were male and $42(33.6 \%)$ of the patients were female. There were $36(28.8 \%)$ patients treated with NACT + IMRT and $89(71.2 \%)$ patients treated with NACT + IMRT + CCRT. The patient distribution between the two different groups was well-balanced based on the prognostic factors, including the age of diagnosis $(P=0.296)$, gender $(P=0.429)$, smoking history $(P=0.162)$, alcohol use $(P=0.454)$, family history $(P=0.525)$, WHO histology $(P=0.603)$, AJCC stage $(P=0.319)$, T stage $(P=0.276), \mathrm{N}$ stage $(P=0.153)$, and courses of NACT $(P=0.548)$.

\section{Treatment-related toxicities}

As shown in Table 4, no grade 5 toxicities were observed during NACT or RT. The frequency of grade 3-4 hematologic toxicities during NACT or RT in NACT + IMRT + CCRT group was lower than that in NACT + IMRT group. Regarding RT, 125 patients completed it.
Regarding NACT, 13 patients (10.4\%) completed one cycle, 93 patients $(74.4 \%)$ completed two cycles, 17 patients (13.6\%) completed three cycles, and 2 patients $(1.6 \%)$ completed four cycles. During NACT, six patients $(4.8 \%)$ received $25 \%$ reduction of dose due to treatment-related toxicities; the most common grade 3-4 hematologic and nonhematologic toxicities were neutropenia $(38,30.4 \%)$, leukopenia $(16,12.8 \%)$, thrombocytopenia $(16,12.8 \%)$, and liver dysfunction $(11,8.8 \%)$.

Regarding CCRT, 20 patients (16.0\%) completed one cycle, 69 patients $(55.2 \%)$ completed two cycles, and 36 patients $(28.8 \%)$ did not receive it. During CCRT, the most common grade 3-4 hematologic and nonhematologic toxicities were oropharyngeal mucositis (18, $14.4 \%)$, nausea/vomiting $(15,12.0 \%)$, thrombocytopenia $(8,6.4 \%)$, and anemia $(7,5.6 \%)$. Of the 36 patients who did not receive CCRT, eleven patients $(30.5 \%)$ suffered grade 3-4 hematologic toxicities at the beginning of RT, nine patients (25\%) suffered grade $3-4$

Table 3 Characteristics of NPC patients treated with NACT + IMRT + CCRT vs NACT + IMRT

\begin{tabular}{|c|c|c|c|}
\hline Total $(n=125)$ & NACT + IMRT $(n=36)$ & NACT + IMRT + CCRT (n=89) & \\
\hline Characteristics & Cases (\%) & Cases (\%) & Pa (Pearson's $\left.\chi^{2}\right)$ \\
\hline Age of diagnosis & & & 0.296 \\
\hline$<55$ & $16(12.8)$ & $46(36.8)$ & \\
\hline$\geq 55$ & $20(16.0)$ & $43(34.4)$ & \\
\hline Gender & & & 0.429 \\
\hline Female & $13(10.4)$ & $29(23.2)$ & \\
\hline Male & $23(18.4)$ & $60(48.0)$ & \\
\hline Smoking history & & & 0.162 \\
\hline Never & $22(17.6)$ & $44(35.2)$ & \\
\hline Ever & $14(11.2)$ & $45(36.0)$ & \\
\hline Alcohol use & & & 0.454 \\
\hline Never & $23(17.2)$ & $58(48.5)$ & \\
\hline Ever & $13(6.1)$ & $31(28.3)$ & \\
\hline Family history & & & 0.525 \\
\hline No & $30(18.4)$ & $57(46.4)$ & \\
\hline Yes & $6(10.4)$ & $32(24.8)$ & \\
\hline WHO histology & & & 0.603 \\
\hline II & $6(4.8)$ & $15(12.0)$ & \\
\hline III & $30(24.0)$ & $74(59.2)$ & \\
\hline AJCC stage & & & 0.319 \\
\hline III & $22(17.6)$ & $60(48.0)$ & \\
\hline$I V A+I V B$ & $14(11.2)$ & $29(23.2)$ & \\
\hline T stage & & & 0.276 \\
\hline TI-2 & II (8.8) & $34(27.2)$ & \\
\hline T3-4 & $25(20.0)$ & $55(44.0)$ & \\
\hline $\mathrm{N}$ stage & & & 0.153 \\
\hline No-I & $17(13.6)$ & $29(23.2)$ & \\
\hline N2-3 & $19(15.2)$ & $60(48.0)$ & \\
\hline Courses of NACT & & & 0.548 \\
\hline I & $4(3.2)$ & $9(7.2)$ & \\
\hline $2-4^{b}$ & $32(25.6)$ & $80(60.0)$ & \\
\hline
\end{tabular}

Notes: ${ }^{\text {aT }}$ wo-sided chi-squared test. ${ }^{\text {} O}$ Only two patients receive four courses of NACT.

Abbreviations: AJCC, American Joint Committee on Cancer; CCRT, concurrent chemotherapy; IMRT, intensity modulated radiotherapy; NACT, neoadjuvant chemotherapy; NPC, nasopharyngeal carcinoma. 
Table 4 Treatment-related acute toxicities during NACT or RT in NPC patients treated with NACT + IMRT + CCRT vs NACT + IMRT

\begin{tabular}{|c|c|c|c|c|c|c|c|c|c|}
\hline \multirow{3}{*}{$\begin{array}{l}\text { During radiotherapy } \\
\text { Acute toxicities }\end{array}$} & \multicolumn{4}{|c|}{ NACT + IMRT $(n=36)$} & \multicolumn{4}{|c|}{ NACT + IMRT + CCRT (n=89) } & \multirow[t]{3}{*}{$P$-value } \\
\hline & \multicolumn{2}{|c|}{ During NACT } & \multicolumn{2}{|c|}{ During radiotherapy } & \multicolumn{2}{|c|}{ During NACT } & \multicolumn{2}{|c|}{ During radiotherapy } & \\
\hline & Grade I-2 & Grade 3-4 & Grade I-2 & Grade 3-4 & Grade I-2 & Grade 3-4 & Grade I-2 & Grade 3-4 & \\
\hline \multicolumn{10}{|l|}{ Hematologic } \\
\hline Leukopenia & 27 (75\%) & 7 (19.4\%) & $20(54.2 \%)$ & $4(11.1 \%)$ & $59(66.3 \%)$ & $9(10.1 \%)$ & $55(61.8 \%)$ & $4(4.5 \%)$ & 0.362 \\
\hline Neutropenia & $15(41.7 \%)$ & $13(36.1 \%)$ & 15 (4I.7\%) & $3(8.3 \%)$ & 40 (44.9\%) & $25(28.1 \%)$ & $27(30.3 \%)$ & $6(6.7 \%)$ & 0.925 \\
\hline Neutropenia fever & I $(2.8 \%)$ & I $(2.8 \%)$ & 0 & 0 & $2(2.2 \%)$ & 0 & $\mathrm{I}(\mathrm{I} .1 \%)$ & 0 & N.A. \\
\hline Thrombocytopenia & $9(25 \%)$ & $7(19.4 \%)$ & $12(33.3 \%)$ & $3(8.3 \%)$ & $18(20.2 \%)$ & $9(10.1 \%)$ & $31(34.8 \%)$ & $5(5.6 \%)$ & 0.702 \\
\hline Anemia & $23(62.5 \%)$ & $5(13.9 \%)$ & $20(54.2 \%)$ & $3(8.3 \%)$ & 58 (65.2\%) & $\mathrm{I}(1.1 \%)$ & $47(52.8 \%)$ & $4(4.5 \%)$ & 0.040 \\
\hline \multicolumn{10}{|l|}{ Nonhematologic } \\
\hline Liver dysfunction & 20 (54.2\%) & $5(13.9 \%)$ & $6(16.7 \%)$ & $2(5.6 \%)$ & 49 (55. I\%) & $6(6.7 \%)$ & 15 (16.9\%) & I (I.I\%) & 0.394 \\
\hline Kidney dysfunction & 0 & 0 & 0 & 0 & 0 & 0 & 0 & 0 & \\
\hline Diarrhea & I (2.8\%) & 0 & 0 & 0 & $2(2.2 \%)$ & 0 & I (I.I\%) & 0 & N.A. \\
\hline Rash & $6(16.7 \%)$ & 0 & I (2.8\%) & 0 & II (I2.4\%) & 0 & $5(5.6 \%)$ & 0 & N.A. \\
\hline Nausea/vomiting & $18(50.0 \%)$ & $4(11.1 \%)$ & $5(13.9 \%)$ & I (2.8\%) & $33(37.1 \%)$ & $6(6.7 \%)$ & 47 (52.8\%) & 14 (I5.7\%) & 0.003 \\
\hline Neurotoxicity & 5 (13.9\%) & 0 & $3(8.3 \%)$ & 0 & 7 (7.9\%) & 0 & $10(10.7 \%)$ & 0 & N.A. \\
\hline $\begin{array}{l}\text { Oropharyngeal } \\
\text { mucositis }\end{array}$ & $3(8.3 \%)$ & 0 & 31 (86.1\%) & $6(16.7 \%)$ & $5(5.6 \%)$ & 0 & $72(80.9 \%)$ & $12(13.5 \%)$ & N.A. \\
\hline Hearing loss & I (2.8\%) & 0 & I (2.8\%) & 0 & $2(2.2 \%)$ & 0 & I (I.I\%) & 0 & N.A. \\
\hline Radiodermatitis & - & - & $6(16.7 \%)$ & I (2.8\%) & - & - & $14(15.7 \%)$ & $4(4.5 \%)$ & N.A. \\
\hline
\end{tabular}

Abbreviations: CCRT, concurrent chemotherapy; IMRT, intensity modulated radiotherapy; N.A., not applicable; NACT, neoadjuvant chemotherapy; NPC, nasopharyngeal carcinoma; RT, radiotherapy.

hematologic toxicities during NACT, accounting for a large proportion of grade 3-4 hematologic toxicities and introducing a bias to higher treatment-related toxicities.

There was significant difference in anemia $(P=0.040)$ and nausea/vomiting $(P=0.003)$ between NACT + IMRT + CCRT group and NACT + IMRT group, while no significance was found in other toxicities between the two different arms.

\section{RT efficacy}

Tumor response in patients following RT is summarized in Table 5. No patient suffered with progressive disease.

For the primary tumor and the neck metastatic nodes, in the NACT + IMRT group, the RR was $91.7 \%$ (33/36), the CR rate was $66.7 \%(24 / 36)$, the PR rate was $25 \%(9 / 36)$, and $8.3 \%(3 / 36)$ of patients showed SD, while the RR was 93.3\% (83/89), the CR rate was $71.9 \%$ (64/89), the PR rate was $21.4 \%(19 / 89)$, and $6.7 \%$ (6/89) of patients showed SD in the NACT + IMRT + CCRT group; in general, the RR was $92.8 \%(116 / 125)$, the CR rate was $70.4 \%(88 / 125)$, the PR rate was $22.4 \%(28 / 125)$, and 9 patients $(7.2 \%)$ showed SD. Furthermore, compared with NACT + IMRT group, patients in NACT + IMRT + CCRT group did not show significantly better RR $(93.3 \%$ vs $91.7 \%, P=1.00)$, CR rate $(71.9 \%$ vs $66.7 \%, P=0.67)$, or PR rate $(21.4 \%$ vs $25 \%, P=0.81)$.

For the primary tumor as well as the neck metastatic nodes, the $\mathrm{RR}, \mathrm{CR}$ rate, $\mathrm{PR}$ rate, and $\mathrm{SD}$ rate were also exhibited; similarly, compared with NACT + IMRT group,

Table 5 Response of the primary site and the neck metastatic nodes in patients treated with NACT + IMRT + CCRT vs NACT + IMRT

\begin{tabular}{|c|c|c|c|c|c|c|c|c|c|}
\hline & \multicolumn{2}{|c|}{ Primary site } & \multirow[b]{2}{*}{$P$-value ${ }^{a}$} & \multicolumn{2}{|l|}{ Neck } & \multirow[b]{2}{*}{$P$-value ${ }^{a}$} & \multicolumn{2}{|c|}{ Primary site + neck } & \multirow[b]{2}{*}{$P_{\text {-value }}{ }^{a}$} \\
\hline & $\begin{array}{l}\text { NACT + } \\
\text { IMRT } \\
(n=36)\end{array}$ & $\begin{array}{l}\text { NACT + } \\
\text { IMRT + CCRT } \\
(n=89)\end{array}$ & & $\begin{array}{l}\text { NACT + } \\
\text { IMRT } \\
(n=36)\end{array}$ & $\begin{array}{l}\text { NACT + } \\
\text { IMRT + CCRT } \\
(n=89)\end{array}$ & & $\begin{array}{l}\text { NACT + } \\
\text { IMRT } \\
(n=36)\end{array}$ & $\begin{array}{l}\text { NACT + IMRT + } \\
\text { CCRT } \\
(n=89)\end{array}$ & \\
\hline$R R(C R+P R)$ & 34 (94.4\%) & 84 (94.4\%) & 0.68 & 35 (97.2\%) & 85 (95.5\%) & 1.00 & 33 (91.7\%) & 83 (93.3\%) & 1.00 \\
\hline $\mathrm{CR}$ & 26 (72.2\%) & 67 (75.3\%) & 0.82 & 30 (83.3\%) & 70 (78.7\%) & 0.63 & 24 (66.7\%) & 64 (7I.9\%) & 0.67 \\
\hline PR & $8(22.2 \%)$ & 17 (19.1\%) & 0.81 & $5(13.9 \%)$ & 15 (I6.8\%) & 0.79 & $9(25.0 \%)$ & $19(21.4 \%)$ & 0.81 \\
\hline SD & $2(5.6 \%)$ & $5(5.6 \%)$ & 0.68 & I (2.8\%) & $4(4.5 \%)$ & 1.00 & $3(8.3 \%)$ & $6(6.7 \%)$ & 1.00 \\
\hline PD & 0 & 0 & - & 0 & 0 & - & 0 & 0 & - \\
\hline
\end{tabular}

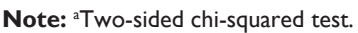

Abbreviations: NACT, neoadjuvant chemotherapy; IMRT, intensity modulated radiotherapy; CCRT, concurrent chemotherapy; RR, response rate; CR, complete response; PR, partial response; SD, stable disease; PD, progressive disease. 
patients in NACT + IMRT + CCRT group did not show significantly better results.

\section{Efficacy and survival}

As shown in Figure 1, in the NACT + IMRT group, patients' 3-year OS, LRFS, DMFS, and PFS rates were 89.4\%, 91.7\%, $83.3 \%$, and $77.8 \%$, respectively, while in the NACT + IMRT + CCRT group, patients' 3-year OS, LRFS, DMFS, and PFS rates were $88.5 \%, 94.4 \%, 82.0 \%$, and $76.4 \%$, respectively; moreover, compared with NACT + IMRT group, $\mathrm{NACT}+\mathrm{IMRT}+\mathrm{CCRT}$ group did not show significant improvements in 3-year OS rate ( $88.5 \%$ vs $89.4 \%, P=0.114)$, LRFS (94.4\% vs 91.7\%, $P=0.124)$, DMFS (83.3\% vs $82.0 \%$, $P=0.668)$, or PFS (77.8\% vs $76.4 \%, P=0.475)$.

During the follow-up period, 8 patients $(6.4 \%)$ suffered from locoregional recurrence and 19 patients (15.2\%) developed distant metastasis, including 4 patients with both locoregional recurrence and distant metastasis. In total, 14 patients were dead, of whom 10 patients were dead from cancer
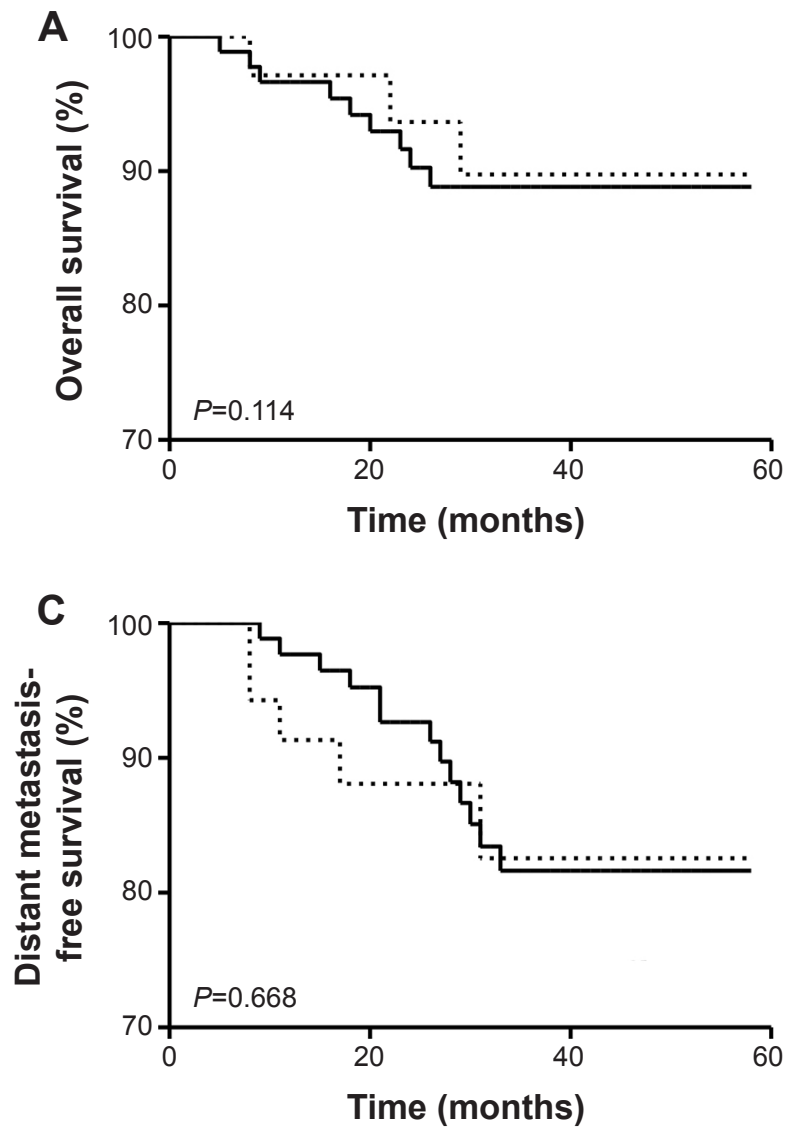

progression, 2 patients were dead from dysphagia following $\mathrm{RT}$, and 2 patients were dead from other diseases.

\section{Prognostic factors}

Univariate analysis with log-rank test and multivariate analysis with the Cox proportion hazards model were used to evaluate the independent potential prognostic factors for OS, DMFS, LRFS, and PFS as shown in Tables 6 and 7.

The potentially important prognostic factors considered in the modeling process were patient age ( $<55$ vs $\geq 55$ years), gender (males vs females), T stage (T1-2 vs T3-4), N stage (N0-1 vs N2-3), use of CCRT (no vs yes), and GTVnx volume $\left(\mathrm{cm}^{3}\right)(<40 \mathrm{vs} \geq 40)$. In the multivariate analysis by prognostic factors, $\mathrm{T}$ classification $(P=0.042)$ and $\mathrm{N}$ classification $(P=0.021)$ were independent prognostic factors for DMFS.

\section{Discussion}

CCRT has been regarded as standard therapeutic approach for locoregionally advanced NPC. ${ }^{17}$ However, distant
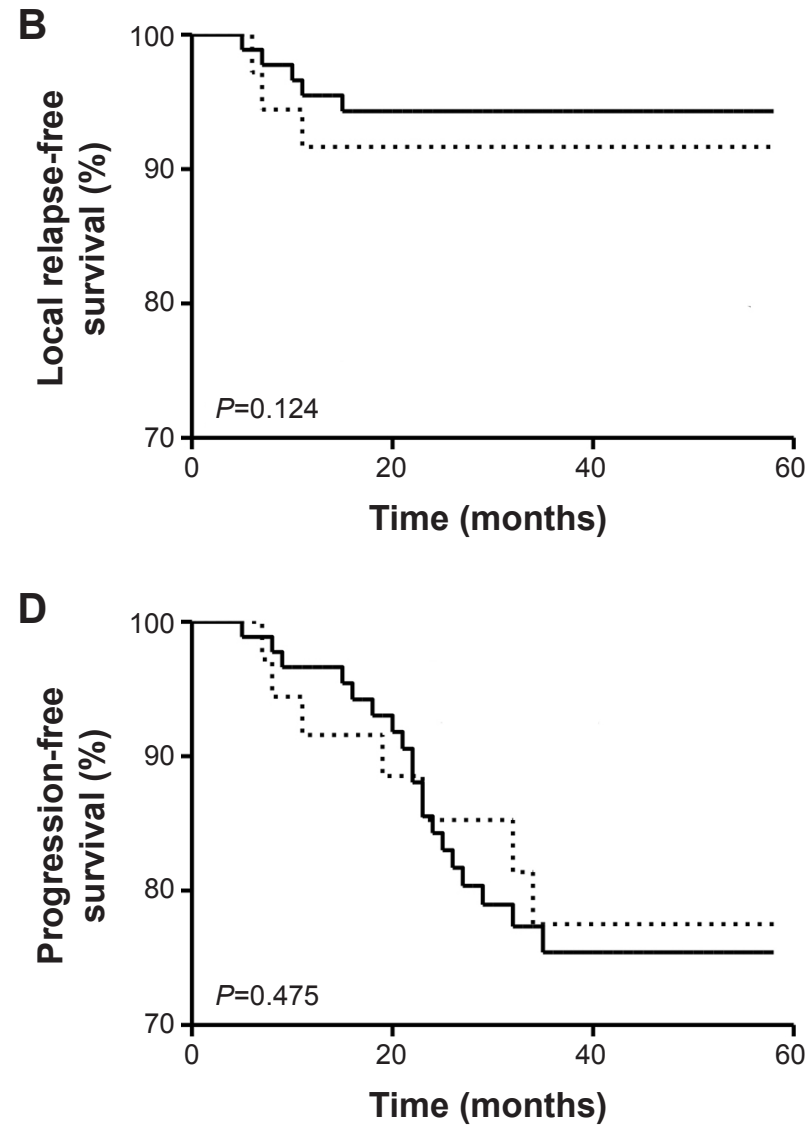

$$
---\mathrm{NACT}+\text { IMRT } \quad \text { - NACT + IMRT + CCRT }
$$

Figure I Survival curves for patients.

Notes: (A) Overall survival (OS), (B) local relapse-free survival (LRFS), (C) distant metastasis-free survival (DMFS), and (D) progression-free survival (PFS) curves for patients in the NACT + IMRT and NACT + IMRT + CCRT groups were exhibited.

Abbreviations: CCRT, concurrent chemotherapy; IMRT, intensity modulated radiotherapy; NACT, neoadjuvant chemotherapy. 
Table 6 Univariate analysis of prognostic factors

\begin{tabular}{|c|c|c|c|c|c|c|c|c|}
\hline \multirow[t]{2}{*}{ Items } & \multicolumn{2}{|c|}{ 3-year OS } & \multicolumn{2}{|c|}{ 3-year LRFS } & \multicolumn{2}{|c|}{ 3-year DMFS } & \multicolumn{2}{|c|}{ 3-year PFS } \\
\hline & $\%$ & $P$-value & $\%$ & $P$-value & $\%$ & $P$-value & $\%$ & $P$-value \\
\hline Age (years) & & 0.872 & & 0.484 & & 0.538 & & 0.347 \\
\hline$<55$ & 89.2 & & 94.7 & & 83.4 & & 77.2 & \\
\hline$\geq 55$ & 87.6 & & 92.8 & & 81.2 & & 75.4 & \\
\hline Gender & & 0.596 & & 0.287 & & 0.773 & & 0.457 \\
\hline Male & 89.3 & & 93.9 & & 83.8 & & 77.5 & \\
\hline Female & 87.7 & & 93.2 & & 81.2 & & 75.8 & \\
\hline$T$ stage & & 0.092 & & $0.005^{*}$ & & 0.574 & & 0.060 \\
\hline TI-2 & 89.8 & & 94.8 & & 82.1 & & 77.9 & \\
\hline T3-4 & 87.5 & & 92.1 & & 82.8 & & 75.8 & \\
\hline$N$ stage & & $0.002^{*}$ & & $0.001 *$ & & $0.032 *$ & & $0.001 *$ \\
\hline N0-I & 92.4 & & 95.6 & & 84.7 & & 78.8 & \\
\hline N2-3 & 83.5 & & 91.8 & & 80.2 & & 74.6 & \\
\hline Smoking & & 0.691 & & 0.554 & & 0.428 & & 0.337 \\
\hline Never & 89.6 & & 91.2 & & 83.5 & & 77.8 & \\
\hline Ever & 86.8 & & 95.4 & & 81.6 & & 76.0 & \\
\hline Alcohol use & & 0.769 & & 0.443 & & 0.352 & & 0.287 \\
\hline Never & 88.2 & & 92.8 & & $8 \mathrm{I} .4$ & & 75.7 & \\
\hline Ever & 89.4 & & 94.5 & & 83.6 & & 77.9 & \\
\hline Use of CCRT & & 0.114 & & 0.124 & & 0.668 & & 0.475 \\
\hline No & 89.4 & & 91.7 & & 83.3 & & 77.8 & \\
\hline Yes & 88.5 & & 94.4 & & 82.0 & & 76.1 & \\
\hline GTVnx volume $\left(\mathrm{cm}^{3}\right)$ & & 0.438 & & 0.473 & & $0.040^{*}$ & & 0.145 \\
\hline$<40$ & 89.1 & & 93.2 & & 84.8 & & 77.5 & \\
\hline$\geq 40$ & 87.7 & & 93.9 & & 80.1 & & 76.2 & \\
\hline
\end{tabular}

Note: $* p<0.05$.

Abbreviations: CCRT, concurrent chemoradiotherapy; DMFS, distant metastasis-free survival; LRFS, local relapse-free survival; OS, overall survival; PFS, progression-free survival.

metastasis still frequently occurred and limited CCRT effect in the era of IMRT..$^{18}$ One previous study indicated that application of greatly decreased the risk of metastasis and prolonged OS time of stage IVb NPC patients treated with IMRT combined with RT or CCRT. ${ }^{19}$ In current retrospective study, we set force to compare the stage III-VIb locoregionally advanced NPC patients' RR, the rates of
PFS, RFS, MFS, and OS between NACT + IMRT group and $\mathrm{NACT}+\mathrm{IMRT}+\mathrm{CCRT}$ group

Clinical trials 21 and 22 and meta-analyses 5 and 6 demonstrated that CCRT leads to improved prognosis in patients. The Intergroup Study 0099 first indicated that CDDP-based CRT improved patients' OS and event-free survival. ${ }^{3}$ In the era of IMRT, however, none of local

Table 7 Multivariate analysis of prognostic factors

\begin{tabular}{|c|c|c|c|c|}
\hline Factors & 3-year OS & 3-year LRFS & 3-year DMFS & 3-year PFS \\
\hline \multicolumn{5}{|l|}{ Age (years) } \\
\hline$<55$ vs $\geq 55$ & 0.572 & 0.483 & 0.396 & 0.424 \\
\hline \multicolumn{5}{|l|}{ Gender } \\
\hline Female vs male & 0.426 & 0.537 & 0.389 & 0.692 \\
\hline \multicolumn{5}{|l|}{ T stage } \\
\hline TI-2 vs T3-4 & 0.224 & 0.186 & $0.042^{*}$ & 0.221 \\
\hline \multicolumn{5}{|l|}{$N$ stage } \\
\hline N0-I vs N2-3 & 0.337 & $0.28 I$ & $0.02 I^{*}$ & 0.342 \\
\hline \multicolumn{5}{|l|}{ Use of CCRT } \\
\hline No vs yes & 0.398 & 0.592 & 0.424 & 0.482 \\
\hline GTVnx volume $\left(\mathrm{cm}^{3}\right.$ & & & & \\
\hline$<40$ vs $\geq 40$ & 0.192 & 0.342 & 0.112 & 0.173 \\
\hline
\end{tabular}

Note: $* P<0.05$.

Abbreviations: CCRT, concurrent chemoradiotherapy; DMFS, distant metastasis-free survival; LRFS, local relapse-free survival; OS, overall survival; PFS, progression-free survival. 
recurrence, distant metastasis rates, and OS after CDDPbased CCRT was improved since the replacement of conventional RT with IMRT. ${ }^{10,23,24}$

To reduce local recurrence and distant metastasis rates, novel CT regimens and optimized sequences for RT and CT delivery require further exploration. GEM was a novel nucleoside antimetabolite that inhibited DNA synthesis. ${ }^{25}$ GEM-based chemotherapy was frequently used during treatment of metastatic NPC patients. ${ }^{26}$ With different structure, NDP exhibited similar mechanism of action compared to CDDP. ${ }^{27}$ Clinically, NDP showed to be more effective with lower frequency of toxicities in comparison with CDDP.${ }^{26}$ Our previous study investigated combination of NDP and GEM as neoadjuvant regimens in locoregionally advanced NPC patients. Unfortunately, their combination showed similar therapeutic effect compared with CBP + GEM or CDDP + GEM. ${ }^{28}$ In patients who received CCRT or NACT followed by radiation, the use of NACT is increasing. ${ }^{29}$ The addition of docetaxel, CDDP, and 5-fluorouracil induction chemotherapy to CCRT was beneficial for the survival of locally advanced NPC with nodal stage N2-3. ${ }^{30}$ The above novel reports published in 2018 and 2017, respectively, showed the significance of addition of NACT in the treatment of locoregionally advanced NPC patients. However, there were also studies indicating the unnecessary addition of NACT; for instance, the induction chemotherapy followed by cetuximab RT is not superior to CCRT for head and neck carcinomas. ${ }^{31}$ Meanwhile, no significant difference in OS or PFS was observed between $\mathrm{IC}+$ volumetric modulated arc therapy group and IC/CCRT group in the treatment of stage II-IVB NPC patients, while more side effects were observed in the IC/CCRT arm. ${ }^{32}$

There was significant difference in anemia and nausea/ vomiting between NACT + IMRT + CCRT group and $\mathrm{NACT}+$ IMRT group, while no significance was found in other toxicities between the two different arms. Patients who did not undergo CCRT showed a higher toxicity, on account of the positive effects of CCRT in the treatment of locoregionally advanced PC. ${ }^{33}$ As for oropharyngeal mucositis, there was no significant difference between $\mathrm{NACT}+\mathrm{IMRT}+\mathrm{CCRT}$ group and NACT + IMRT group, which might due to the application of IMRT, consistent with a previous study indicating that texture analysis as a predictor of RT induced xerostomia in head and neck patients undergoing IMRT. ${ }^{34}$

In the current study, we sought to investigate whether NDP-based CCRT could improve prognosis of patients receiving NACT + IMRT. In terms of tumor response at the primary site and neck metastatic nodes following RT,
RR in the NACT + IMRT + CCRT group was as high as 93.3\%, which was not significantly higher than that in the NACT + IMRT group (91.7\%, $P=1.000)$. The CR rate in $\mathrm{NACT}+\mathrm{IMRT}+\mathrm{CCRT}$ group was not superior to NACT + IMRT group $(71.9 \%$ vs $66.70 \%, P=0.666)$. For patients in the NACT + IMRT + CCRT group, the 3-year OS, LRFS, DMFS, and PFS rates were 88.5\%, 94.4\%, 82.0\%, and $76.4 \%$, respectively, which were not higher than those in the NACT + IMRT group that had an OS rate of $89.4 \%$ $(P=0.114)$, DMFS rate of $83.3 \%(P=0.668)$, LRFS rate of $91.7 \%(P=0.124)$, and $P F S$ rate of $77.8 \%(P=0.475)$. In summary, the results did not demonstrate any benefit for survival when NDP-based CCRT was added to IMRT following NACT regimen (NDP + GEM), which are in agreement with previous comparisons between IMRT with or without $\mathrm{CT},{ }^{10,22}$ while inconsistent with results from Ji et al. ${ }^{11}$

In the study by Cao et al, ${ }^{10} 180$ nonmetastatic T4 NPC patients, of whom 117 were treated by CCRT + IMRT and 63 were treated by IMRT, were retrospectively analyzed with a median follow-up time of 58.97 months. There was no statistically significant difference between CCRT + IMRT group and IMRT group regarding the 3/5-year local failurefree survival rates $(89.2 \%$ vs $85.9 \%)$, regional failure-free survival rates (94.4\% vs $94.4 \%$ ), distant failure-free survival rates $(79.9 \%$ vs $76.2 \%)$, or OS rates $(78.9 \%$ vs $65.3 \%)$. In the study by Sun et al, 868 nonmetastatic NPC patients who were treated by IMRT with a median follow-up time of 50 months were retrospectively analyzed, of whom 217 received additional CCRT. The 5-year disease specific survival (78.4\% vs $79.1 \%, P=0.340)$, LRFS (89.7\% vs $89.1 \%, P=0.197)$, RRFS (96.6\% vs $96.0 \%, P=0.280)$, DMFS $(79.0 \%$ vs $80.8 \%$, $P=0.998)$, and PFS rates $(70.5 \%$ vs $68.8 \%, P=0.480)$ were similar in IMRT + CCRT group vs IMRT group. ${ }^{22}$

In the study by Ji et al, 276 patients at stage II-IVb NPC with a median follow-up time of 33.8 months were analyzed. Compared with the IMRT group, addition of CCRT or NACT to IMRT is available to prolong patients' DFS $(P=0.03)$ or RFS $(P=0.01)$ and DFS $(P=0.03)$, respectively, with no significant benefit in OS, MFS, RFS, or DFS. ${ }^{11}$

Based on the RR and 3-year OS rate in the NACT + IMRT group and NACT + IMRT + CCRT group, it appears that it is not necessary to add NDP-based CCRT to IMRT in patients with locoregionally advanced NPC who have received NACT regimen (NDP + GEM). Whereas there are several limitations in our retrospective study (1) the median follow-up period was insufficient; 2) the low numerosity of the cohort analyzed; $3)$ the proportion of patients who had a poorer prognosis at N2-3 was higher in NACT + IMRT + CCRT group; 
4) the disequilibrium between the arms, as the proportion of patients with more significant health issues and a less advanced N stage was higher in the NACT + IMRT group; 5) 20 patients completed only one cycle of CCRT), it is unclear whether the number of cycles of CCRT influences the efficacy when combined with IMRT. Therefore, larger and better-designed studies with well-balanced prognostic factors with an adequate follow-up period were required to confirm the efficacy of CCRT + IMRT in locoregionally advanced NPC patients.

\section{Conclusion}

No significant difference was observed in combined RR, CR, LRFS, DMFS, PFS, or OS rates between the two groups. Therefore, it might be unnecessary to add CCRT to IMRT following NACT in locoregionally advanced NPC in clinical.

\section{Acknowledgment}

The authors thank Elixigen Co. for editing this article prior to submission.

\section{Disclosure}

The authors report no conflicts of interest in this work.

\section{References}

1. Wei KR, Zheng RS, Zhang SW, Liang ZH, Ou ZX, Chen WQ. Nasopharyngeal carcinoma incidence and mortality in China in 2010. Chin J Cancer. 2014;33(8):381-387.

2. Vokes EE, Liebowitz DN, Weichselbaum RR. Nasopharyngeal carcinoma. Lancet. 1997;350(9084):1087-1091.

3. Chan AT, Leung SF, Ngan RK, et al. Overall survival after concurrent cisplatin-radiotherapy compared with radiotherapy alone in locoregionally advanced nasopharyngeal carcinoma. $J$ Natl Cancer Inst. 2005;97(7): 536-539.

4. Al-Sarraf M, LeBlanc M, Giri PG, et al. Chemoradiotherapy versus radiotherapy in patients with advanced nasopharyngeal cancer: phase III randomized Intergroup study 0099. J Clin Oncol. 1998;16(4):1310-1317.

5. Langendijk JA, Leemans CR, Buter J, Berkhof J, Slotman BJ. The additional value of chemotherapy to radiotherapy in locally advanced nasopharyngeal carcinoma: a meta-analysis of the published literature. J Clin Oncol. 2004;22(22):4604-4612.

6. Baujat B, Audry H, Bourhis J, et al; MAC-NPC Collaborative Group. Chemotherapy in locally advanced nasopharyngeal carcinoma: an individual patient data meta-analysis of eight randomized trials and 1753 patients. Int J Radiat Oncol Biol Phys. 2006;64(1):47-56.

7. Lu H, Yao M. The current status of intensity-modulated radiation therapy in the treatment of nasopharyngeal carcinoma. Cancer Treat Rev. 2008;34(1):27-36.

8. Lai SZ, Li WF, Chen L, et al. How does intensity-modulated radiotherapy versus conventional two-dimensional radiotherapy influence the treatment results in nasopharyngeal carcinoma patients? Int J Radiat Oncol Biol Phys. 2011;80(3):661-668.

9. Geara FB, Sanguineti G, Tucker SL, et al. Carcinoma of the nasopharynx treated by radiotherapy alone: determinants of distant metastasis and survival. Radiother Oncol. 1997;43(1):53-61.

10. Cao CN, Luo JW, Gao L, et al. Concurrent chemotherapy for T4 classification nasopharyngeal carcinoma in the era of intensity-modulated radiotherapy. PLoS One. 2015;10(3):e0119101.
11. Ji X, Xie C, Hu D, Fan X, Zhou Y, Zheng Y. Survival benefit of adding chemotherapy to intensity modulated radiation in patients with locoregionally advanced nasopharyngeal carcinoma. PLoS One. 2013;8(2):e56208.

12. Ekenel M, Keskin S, Basaran M, et al. Induction chemotherapy with docetaxel and cisplatin is highly effective for locally advanced nasopharyngeal carcinoma. Oral Oncol. 2011;47(7):660-664.

13. Yau TK, Lee AW, Wong DH, et al. Induction chemotherapy with cisplatin and gemcitabine followed by accelerated radiotherapy and concurrent cisplatin in patients with stage IV(A-B) nasopharyngeal carcinoma. Head Neck. 2006;28(10):880-887.

14. Hui EP, Ma BB, Leung SF, et al. Randomized phase II trial of concurrent cisplatin-radiotherapy with or without neoadjuvant docetaxel and cisplatin in advanced nasopharyngeal carcinoma. J Clin Oncol. 2009; 27(2):242-249.

15. Ou Yang PY, Xie C, Mao YP, et al. Significant efficacies of neoadjuvant and adjuvant chemotherapy for nasopharyngeal carcinoma by metaanalysis of published literature-based randomized, controlled trials. Ann Oncol. 2013;24(8):2136-2146.

16. Zheng J, Wang G, Yang GY, et al. Induction chemotherapy with nedaplatin with 5-FU followed by intensity-modulated radiotherapy concurrent with chemotherapy for locoregionally advanced nasopharyngeal carcinoma. Jpn J Clin Oncol. 2010;40(5):425-431.

17. Chen Y, Liu MZ, Liang SB, et al. Preliminary results of a prospective randomized trial comparing concurrent chemoradiotherapy plus adjuvant chemotherapy with radiotherapy alone in patients with locoregionally advanced nasopharyngeal carcinoma in endemic regions of China. Int J Radiat Oncol Biol Phys. 2008;71(5):1356-1364.

18. Chen X, Lei H, Liang Z, Li L, Qu S, Zhu X. Intensity-modulated radiotherapy controls nasopharyngeal carcinoma distant metastasis and improves survival of patients. Springerplus. 2016;5(1):1459.

19. Xu T, Shen C, Ou X, He X, Ying H, Hu C. The role of adjuvant chemotherapy in nasopharyngeal carcinoma with bulky neck lymph nodes in the era of IMRT. Oncotarget. 2016;7(15):21013-21022.

20. Salama JK, Haddad RI, Kies MS, et al. Clinical practice guidance for radiotherapy planning after induction chemotherapy in locoregionally advanced head-and-neck cancer. Int J Radiat Oncol Biol Phys. 2009; 75(3):725-733.

21. Chan AT, Teo PM, Ngan RK, et al. Concurrent chemotherapyradiotherapy compared with radiotherapy alone in locoregionally advanced nasopharyngeal carcinoma: progression-free survival analysis of a phase III randomized trial. J Clin Oncol. 2002;20(8):2038-2044.

22. Lin JC, Jan JS, Hsu CY, Liang WM, Jiang RS, Wang WY. Phase III study of concurrent chemoradiotherapy versus radiotherapy alone for advanced nasopharyngeal carcinoma: positive effect on overall and progression-free survival. J Clin Oncol. 2003;21(4):631-637.

23. Sun X, Su S, Chen C, et al. Long-term outcomes of intensity-modulated radiotherapy for 868 patients with nasopharyngeal carcinoma: an analysis of survival and treatment toxicities. Radiother Oncol. 2014;110(3):398-403.

24. Lin S, Pan J, Han L, et al. Update report of nasopharyngeal carcinoma treated with reduced-volume intensity-modulated radiation therapy and hypothesis of the optimal margin. Radiother Oncol. 2014;110(3): 385-389.

25. Barton-Burke M. Gemcitabine: a pharmacologic and clinical overview. Cancer Nurs. 1999;22(2):176-183.

26. Leong SS, Wee J, Tay MH, et al. Paclitaxel, carboplatin, and gemcitabine in metastatic nasopharyngeal carcinoma: a Phase II trial using a triplet combination. Cancer. 2005;103(3):569-575.

27. Kurita H, Yamamoto E, Nozaki S, Wada S, Furuta I, Kurashina K. Multicenter phase I trial of induction chemotherapy with docetaxel and nedaplatin for oral squamous cell carcinoma. Oral Oncol. 2004;40(10):1000-1006.

28. Jin T, Chen XZ, Liu JJ. Evaluation of the efficacy and safety of a neoadjuvant gemcitabine and nedaplatin regimen followed by radiotherapy or concurrent chemoradiotherapy in locoregionally advanced nasopharyngeal carcinoma. Oncol Lett. 2015;10(2):1123-1130.

29. Tam M, Lee A, Wu SP, et al. Neoadjuvant chemotherapy in localregionally advanced nasopharyngeal carcinoma: A National Cancer Database analysis. Laryngoscope. Epub 2018 Aug 22. 
30. Kawahira M, Yokota T, Hamauchi S, et al. Survival benefit of adding docetaxel, cisplatin, and 5-fluorouracil induction chemotherapy to concurrent chemoradiotherapy for locally advanced nasopharyngeal carcinoma with nodal Stage N2-3. Jpn J Clin Oncol. 2017;47(8):705-712.

31. Geoffrois L, Martin L, De Raucourt D, et al. Induction chemotherapy followed by cetuximab radiotherapy is not superior to concurrent chemoradiotherapy for head and neck carcinomas: results of the GORTEC 2007-02 phase III randomized trial. Epub 2018 Jul 17.

32. Liu L, Fei Z, Chen M, et al. Induction chemotherapy plus concurrent chemoradiotherapy versus induction chemotherapy plus volumetric modulated arc therapy alone in the treatment of stage II-IVB nasopharyngeal carcinoma patients: a retrospective controlled study. Radiat Oncol. 2018;13(1):148.
33. Kong M, Lim YJ, Kim Y. Concurrent chemoradiotherapy for loco-regionally advanced nasopharyngeal carcinoma: treatment outcomes and prognostic factors. Asian Pac J Cancer Prev. 2018;19(6): 1591-1599.

34. Nardone V, Tini P, Nioche $\mathrm{C}$, et al. Texture analysis as a predictor of radiation-induced xerostomia in head and neck patients undergoing IMRT. Radiol Med. 2018;123(6):415-423.

\section{Publish your work in this journal}

OncoTargets and Therapy is an international, peer-reviewed, open access journal focusing on the pathological basis of all cancers, potential targets for therapy and treatment protocols employed to improve the management of cancer patients. The journal also focuses on the impact of management programs and new therapeutic agents and protocols on

\section{Dovepress}

patient perspectives such as quality of life, adherence and satisfaction The manuscript management system is completely online and includes a very quick and fair peer-review system, which is all easy to use. Visit http://www.dovepress.com/testimonials.php to read real quotes from published authors. 